\title{
Loss of H2A.Z Is Not Sufficient to Determine Transcriptional Activity of Snf2-Related CBP Activator Protein or p400 Complexes
}

\author{
Tamara A. Bowman, ${ }^{1}$ Madeline M. Wong, ${ }^{2}$ Linda K. Cox, ${ }^{1}$ \\ Joseph J. Baldassare, ${ }^{1}$ and John C. Chrivia ${ }^{1}$ \\ ${ }^{1}$ Department of Pharmacological and Physiological Science, Saint Louis University School of Medicine, 1402 South Grand Boulevard, \\ Saint Louis, MO 63104, USA \\ ${ }^{2}$ Laboratory of Receptor Biology and Gene Expression, National Cancer Institute, National Institute of Health Bethesda, \\ MD 20892, USA
}

Correspondence should be addressed to John C. Chrivia, chrivia@slu.edu

Received 11 January 2011; Accepted 1 April 2011

Academic Editor: J. R. Davie

Copyright ( 2011 Tamara A. Bowman et al. This is an open access article distributed under the Creative Commons Attribution License, which permits unrestricted use, distribution, and reproduction in any medium, provided the original work is properly cited.

The p400 and SRCAP (Snf2-related CBP activator protein) complexes remodel chromatin by catalyzing deposition of histone H2A.Z into nucleosomes. This remodeling activity has been proposed as a basis for regulation of transcription by these complexes. Transcript levels of $p 21$ or Sp1 mRNAs after knockdown of p400 or SRCAP reveals that each regulates transcription of these promoters differently. In this study, we asked whether deposition of H2A.Z within specific nucleosomes by p400 or SRCAP dictates transcriptional activity. Our data indicates that nucleosome density at specific $p 21$ or Sp1 promoter positions is not altered by the loss of either remodeling complex. However, knockdown of SRCAP or p400 reduces deposition of H2A.Z 50\% into all p21 and Sp1 promoter nucleosomes. Thus, H2A.Z deposition is not targeted to specific nucleosomes. These results indicate that the deposition of H2A.Z by the p400 or SRCAP complexes is not sufficient to determine how each regulates transcription. This conclusion is further supported by studies that demonstrate a SRCAP $\triangle A T P$ mutant unable to deposit H2A.Z has similar transcriptional activity as wild-type SRCAP.

\section{Introduction}

The histone variant H2A.Z has been shown to have multiple functions in mammalian cells. It is essential for embryonic development, proper segregation of chromosomes $[1,2]$, and a number of studies indicate it plays a role in both activation and repression of transcription [3-5]. Aberrant H2A.Z expression may also play a role in some human diseases, since it has been demonstrated to play a role in cardiac hypertrophy [6] and H2A.Z levels are elevated in breast cancer [7, 8].

Recent studies have examined the genomewide distribution of H2A.Z in human cells. These studies found that nucleosomes located in promoter regions are highly enriched in H2A.Z, indicating a positive correlation that exists between gene activity and H2A.Z deposition $[9,10]$. The deposition of H2A.Z at these sites has been hypothesized to promote organization of nucleosomes at the promoter providing the optimal architecture for activation of transcription. How incorporation of H2A.Z into nucleosomes functions to increase promoter organization and contribute to regulation of transcription is not clear. Several studies indicate that nucleosomes comprised of recombinant H2A.Z or native chicken erythrocyte H2A.Z are more stable than $\mathrm{H} 2 \mathrm{~A}$-containing nucleosomes $[11,12]$. Other studies, however, have raised the possibility that in certain circumstances, when nucleosomes also contain the histone variant H3.3, H2A.Z decreases nucleosome stability $[13,14]$. These studies remain controversial since in vitro studies have reported no differences that exist in the stability of nucleosomes containing H3.3/H2A.Z compared to those containing $\mathrm{H} 3.3 / \mathrm{H} 2 \mathrm{~A}$ [15].

In mammalian cells, SRCAP and p400 are the catalytic subunits of larger complexes that have been demonstrated in vitro and in vivo to deposit H2A.Z into nucleosomes [3, $5,16]$. A comparison of the structure of p400 and SRCAP 
indicates they share a conserved bipartite ATPase domain and a HSA domain. They also have distinct domains: SRCAP contains multiple $\mathrm{A} / \mathrm{T}$ hook domains in the $\mathrm{C}$ terminal end, whereas $\mathrm{p} 400$ has a SANT domain. The human SRCAP complex contains ten subunits (SRCAP, DMAP1, BAF53a, ARP6, Gas41, Tip49a, Tip49b, ZnF-HIT1, YL1, and H2A.Z). The p400 complex shares some of these subunits (DMAP1, BAF53a, ARP6, Gas41, Tip49a, Tip49b, and YL1) but contains additional unique subunits (TRRAP, p400, TRCp120, EPC, EPC-like, TIP60, ING3, MRG15, MRGX, and MRGBP FLJ11730) [17, 18]. The presence of two complexes with H2A.Z deposition activity is intriguing and raises the possibility that they are targeted to different sites within the same promoter or to different promoters. Targeting of the p400 complex has been studied; p400 appears to be recruited to sites that bind p53 or c-Myc [5]. Specific sequences which recruit the SRCAP complex to promoters have not been established, however, interaction of SRCAP with CBP may allow targeting to a variety of promoters [19]. One outcome of targeting of the p400 and SRCAP complexes to different sites within promoters is that they may direct deposition of H2A.Z into distinct subpopulations of nucleosomes and, as a consequence, have different effects on transcription. In support of this hypothesis, recent studies on the $p 21$ promoter suggest that while knockdown of SRCAP or $\mathrm{p} 400$ expression disrupts H2A.Z deposition, only the loss of p400 results in activation of transcription [5].

In this report, we test the hypothesis that the p400 and SRCAP complexes play distinct roles in regulating transcription of the p21 promoter through deposition of H2A.Z into distinct populations of nucleosomes. For these studies, we measure the density of H2A.Z-containing nucleosomes within the $p 21$ promoter and ask whether knockdown of p400 or SRCAP expression disrupts H2A.Z deposition into nucleosomes at specific locations. The results of these studies indicate the loss of p400 or SRCAP results in equivalent changes in $\mathrm{H} 2 \mathrm{~A} . \mathrm{Z}$ binding at all nucleosomes, suggesting a non-H2A.Z-related activity associated with the p400 complex is critical to its ability to repress transcription of the $p 21$ promoter [20]. Studies with the Sp1 promoter demonstrate that knockdown of p400 decreases H2A.Z deposition at all nucleosomes but did not decrease transcription. In contrast, knockdown of SRCAP decreases H2A.Z deposition at most, but not all, nucleosomes, yet transcription decreases. Thus, non-H2A.Z-related activity associated with the SRCAP complex is critical for activation of transcription at the SpI promoter. Collectively, these studies indicate that H2A.Z deposition activity by the p400 and SRCAP complexes is not sufficient to explain how they regulate transcription of the $S p 1$ and $p 21$ promoters.

\section{Results}

Previous studies in U2OS cells indicated that knockdown of p400 and SRCAP results in equivalent loss of H2A.Z deposition [5] at the $p 21$ promoter. Surprisingly, loss of p400 expression resulted in activation of transcription of the p21 promoter whereas knockdown of SRCAP had no effect [5]. We subsequently confirmed these results in the lung adenocarcinoma A549 cell line (Figures 3(b) and $\mathrm{S} 1$ of the supplementary material available online at doi:10.1155/2001/715642). To understand how the p400 and SRCAP complexes differentially regulate transcription of the p21 promoter we carried out a series of experiments to determine if they deposit H2A.Z into distinct nucleosomes at distinct locations within the $p 21$ promoter.

Our initial studies were carried out to measure the density of nucleosomes at the $p 21$ promoter. For these studies, chromatin was cross-linked by treatment with formaldehyde and extensively digested by treatment with micrococcal nuclease.

Nondigested chromatin was removed by centrifugation and the supernatant containing released chromatin layered on a sucrose density gradient to allow separation of mononucleosomes from higher-order nucleosomes. Analysis of the fractions from the sucrose density gradient for DNA and histone $\mathrm{H} 3$ content validates that the preparation contains a single population of nucleosomes with DNA 147 base pairs (bp) in size (supplemental Figure S2). This indicates that our protocol generates a pool of mononucleosomes that serves as a source of DNA to measure the nucleosomes within the $p 21$ promoter. For these experiments fourteen primer sets were designed that flanked the p400-binding site in the $p 21$ promoter that was previously characterized as overlapping the site of highest deposition of H2A.Z and the major binding site of p53 [5]. The design of the primer sets ensures amplification of overlapping regions smaller than an individual nucleosome.

The results of this approach indicate that the regions of highest mononucleosome DNA density overlaps the same DNA sequence where the highest p400 binding was observed in U2OS cells, from approximately-2668 to-2092 bp upstream of the transcription start site (TSS) (Figure 1(a), black bars). To ask if the nucleosomes are repositioned in the absence of SRCAP or p400, the expression of each protein was reduced by siRNA treatment. Knockdown of SRCAP or p400 was confirmed by Western blot (Figure 1(b)) and did not significantly alter nucleosome density at any position (open and gray bars in Figure 1(a)).

To determine which nucleosomes contain H2A.Z, nucleosome ChIP assays were performed using anti-H2AZ antibodies. The result of these experiments indicates that H2A.Z is not deposited into specific nucleosomes but rather is broadly distributed into all the nucleosomes adjacent to the p400: binding site in the $p 21$ promoter (Figure 2(a), black bars). Knockdown of either SRCAP or p400 expression results in $\sim 50 \%$ decrease in deposition of H2A.Z into most nucleosomes (open and gray bars in Figure 2(a)). This was not due to an indirect affect, for example, decreased cellular level of H2A.Z, but due rather to loss of deposition by $\mathrm{p} 400$ and SRCAP (Figure 2(b)).

To determine whether H2A.Z plays a similar role at other promoters, we also examined H2A.Z deposition into nucleosomes at the Sp1 promoter. This promoter was chosen because knockdown of SRCAP and p400 affects transcription of $S p 1$ differently than $p 21$. At the $S p 1$ promoter, knockdown of SRCAP decreases transcription [3], whereas loss of p400 


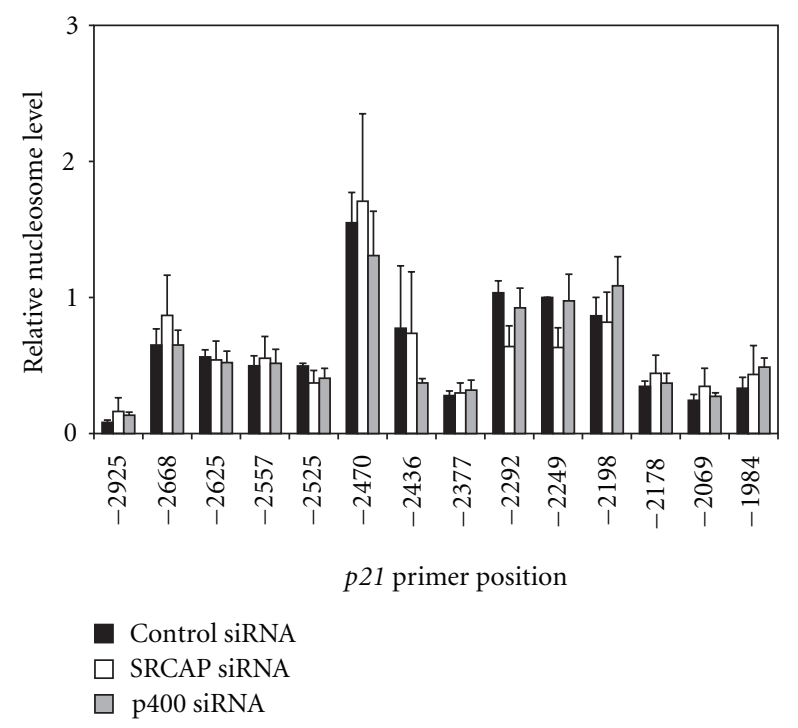

(a)

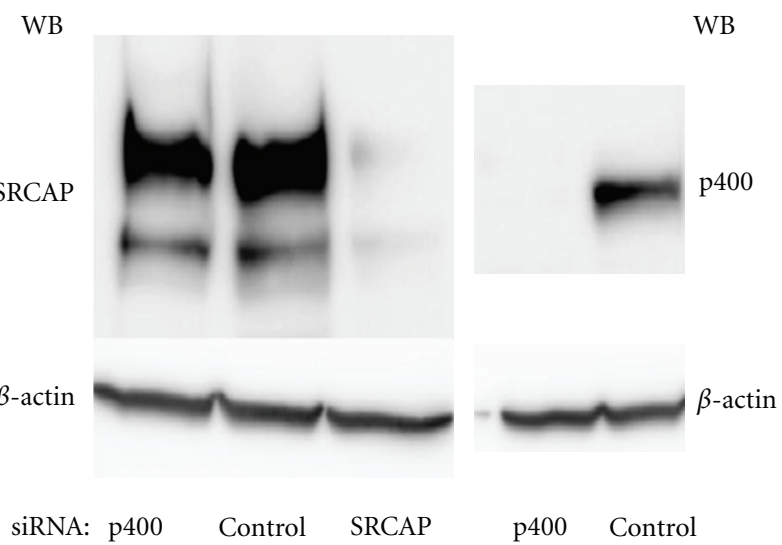

(b)

FIgURE 1: The density of nucleosomes at the $p 21$ promoter nucleosome is not altered in the absence of SRCAP or p400. A549 cells were transfected with control, SRCAP or p400 siRNA and harvested 72 hours later. In (a), DNA was isolated from mononucleosomes and amplified by qPCR using overlapping primers tiling the $p 21$ promoter (see Table S1(c)) and presented relative to the amount of DNA amplified at position -2249 . The graph represents the mean result and standard error of three or more independent ChIP experiments. In (b), knockdown of SRCAP or p400 protein, compared to control-transfected cells, was confirmed by Western blot. Beta actin was used as a loading control.

has no effect on transcription (Figure 3). To measure nucleosome density, sixteen primer sets were designed, flanking the SRCAP-binding site in the Sp1 promoter that was previously characterized [3]. Examination of the Sp1 promoter indicates that it contains nucleosomes at several positions including directly downstream of the TSS (Figure 4, black bars). This result is consistent with the findings of a recent genomewide survey of human promoters that indicate that several strongly phased nucleosomes flank the TSS of most expressed genes [10]. The Sp1 promoter, however, also contains a large nucleosome free region (NFR) at -788 to

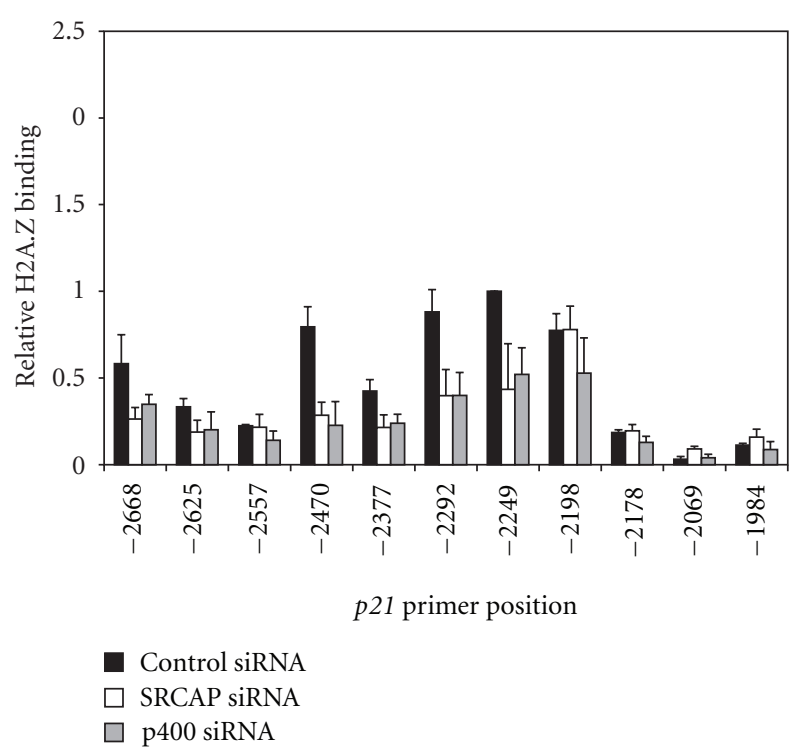

(a)

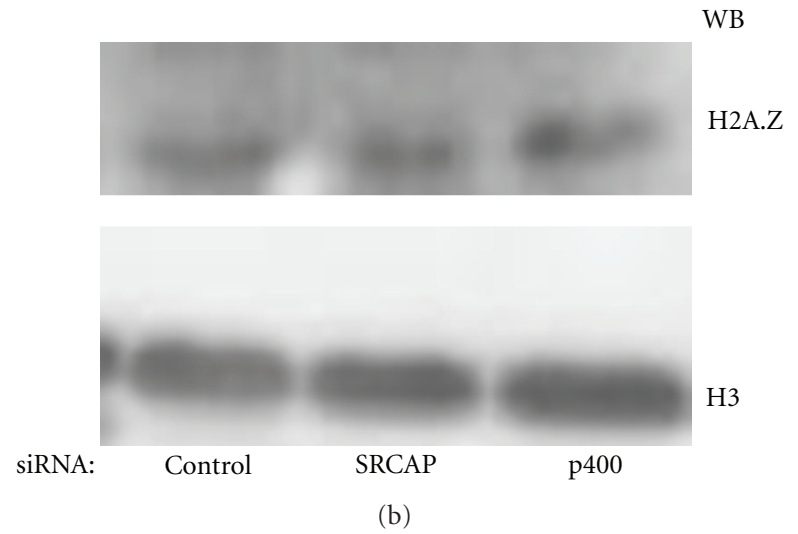

Figure 2: Knockdown of SRCAP or p400 decreases H2A.Z deposition into $p 21$ promoter nucleosomes. A549 cells were transfected with control, SRCAP or p400 siRNA and harvested 72 hours later. In (a), nucleosome ChIP assays were performed using anti-H2A.Z antibody and immunoprecipitated DNA was amplified by qPCR using the indicated primer sets (see Table S1(c)). The amount of DNA amplified at each position is presented relative to the amount of DNA amplified at position -2249. The graph represents the mean result and standard error of three or more independent ChIP experiments. In (b), histones were acid-extracted and protein levels were determined by Western blot analysis using anti-H2A.Z antibody. Histone $\mathrm{H} 3$ was used as a loading control.

-60, where SRCAP binds the promoter. Knockdown of p400 or SRCAP expression did not alter formation of the NFR nor did it alter the density of nucleosomes at any position (gray and open bars in Figure 4).

Nucleosomes flanking the NFR in the Sp1 promoter contained H2A.Z (Figure 5, black bars) as found at other eukaryotic gene promoters $[14,21,22]$. Knockdown of p400 or SRCAP expression did not cause depletion of H2A.Z at specific nucleosomes (gray and open bars in Figure 5), but as was observed with the $p 21$ promoter, p400 and SRCAP appear to have equivalent roles in maintaining normal levels 


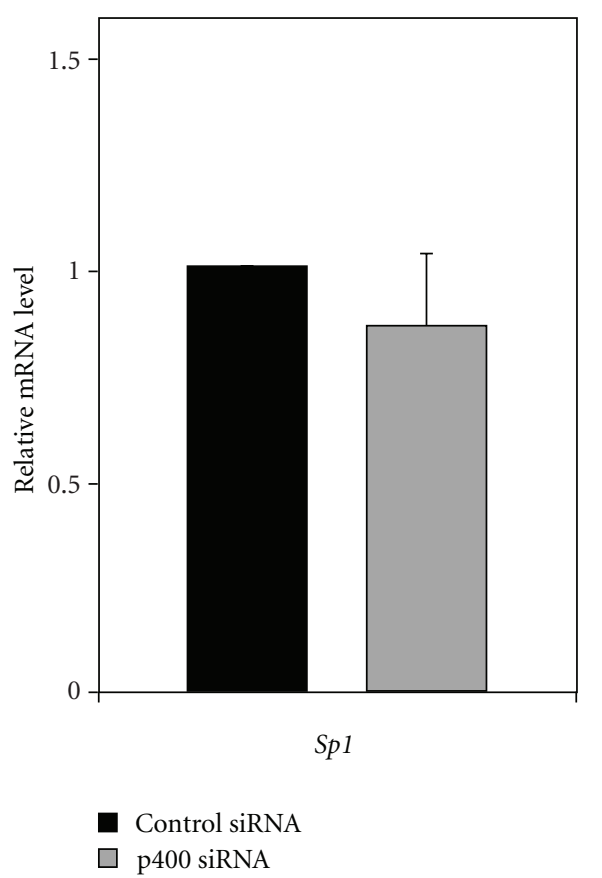

(a)

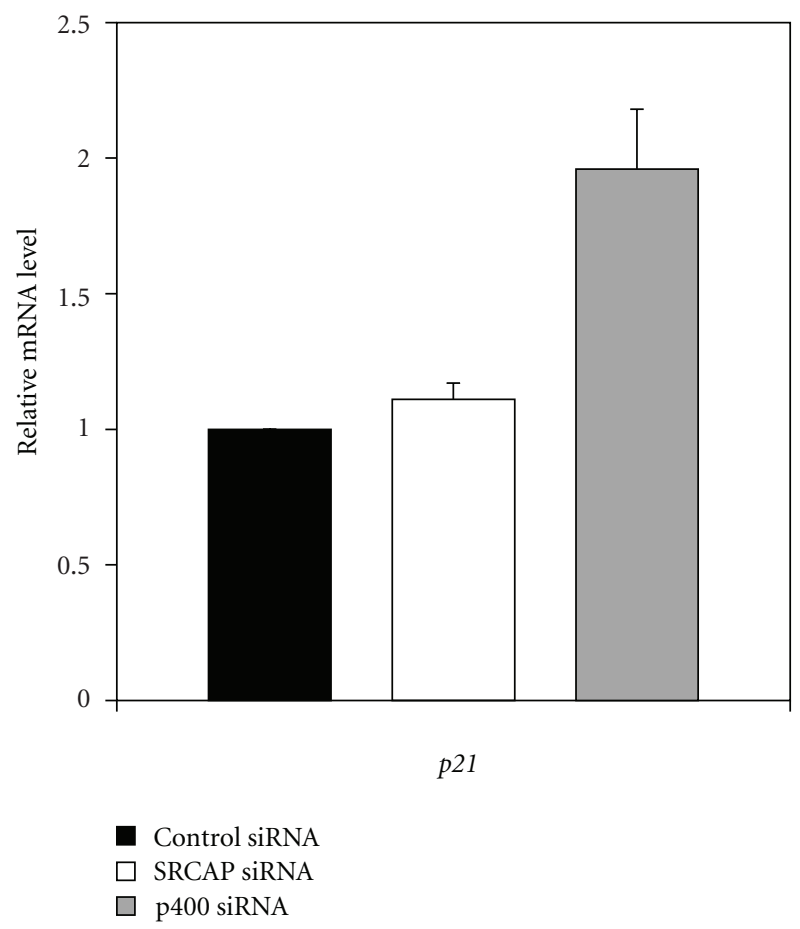

(b)

Figure 3: Knockdown of p400 and SRCAP expression differently regulates transcription of the $S p 1$ promoter and $p 21$ promoter. A549 cells were transfected where indicated with control, SRCAP or p400 siRNA, harvested 72 hours later and total RNA isolated. In (a), the level of $S p 1$ mRNA was assessed using RT-qPCR using primers listed in [3]. In (b), the level of $p 21$ mRNA was determined by RT-qPCR using primers listed in Table S1(a). The graphs show the mean result and standard error from three experiments.

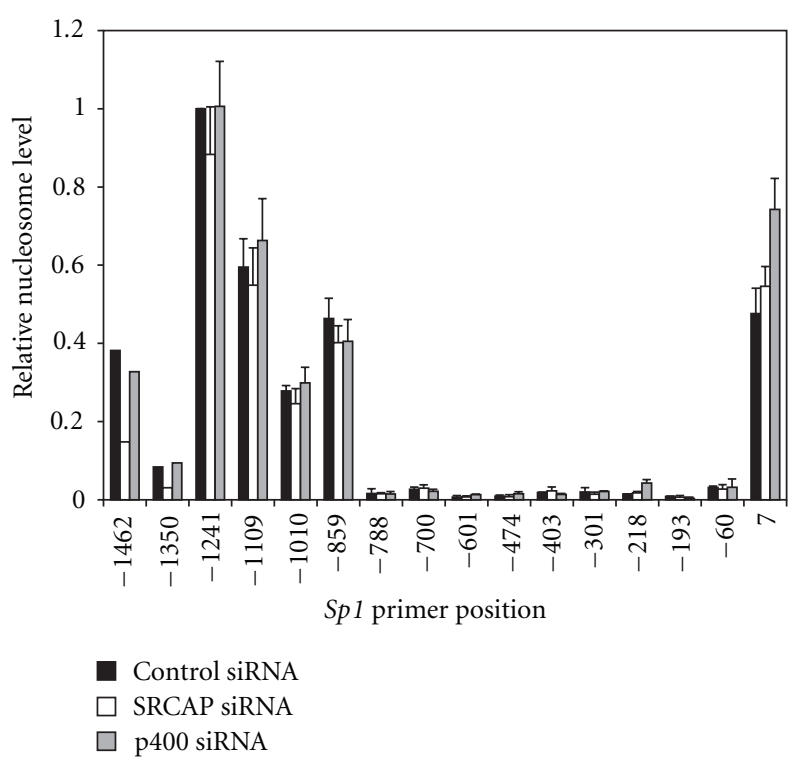

Figure 4: The density of nucleosomes at the $S p 1$ promoter is not altered in the absence of SRCAP or p400. A549 cells were transfected with control, SRCAP or p400 siRNA and harvested 72 hours later. DNA was isolated from mononucleosomes and amplified by qPCR using overlapping primer sets tiling the $S p 1$ promoter (see Table S2) and presented relative to the amount of DNA amplified at position -1241 . The graph represents the mean result and standard error of three or more independent ChIP experiments.

of H2A.Z deposition into all nucleosomes except at positions -1010 and -700 . At these positions, p400, but not SRCAP, regulates deposition of H2A.Z, indicating specificity in the selection of nucleosomes targeted by the SRCAP complex for deposition of H2A.Z.

This suggests that retention of H2A.Z deposition at specific nucleosomes following knockdown of SRCAP, but not p400, may cause repression of transcription. A more likely explanation, and one we favor, is that the ability of the SRCAP complex to regulate transcription at the Sp1 promoter must be determined by non-H2A.Z-related activities absent in the p400 complex. To test for the presence of nonH2A.Z-related transcriptional activity of SRCAP, a mutant unable to bind ATP (K689R, SRCAP $\left.{ }_{\triangle A T P}\right)$ [23] and deposit H2A.Z [16] was tested for the ability to activate transcription. The result of this experiment (Figure 6) indicates that the $\mathrm{SRCAP}_{\triangle \mathrm{ATP}}$ mutant has similar transcriptional activity as wild-type SRCAP. This indicates that SRCAP has the ability to activate transcription independent of the ability to deposit H2A.Z.

\section{Discussion}

The ability of the SRCAP and p400 complexes to deposit the histone variant $\mathrm{H} 2 \mathrm{~A}$. Z into nucleosomes has been previously established in vitro using highly purified complexes. The physiological relevance of this activity in vivo has been confirmed by ChIP assays that demonstrate that knockdown 


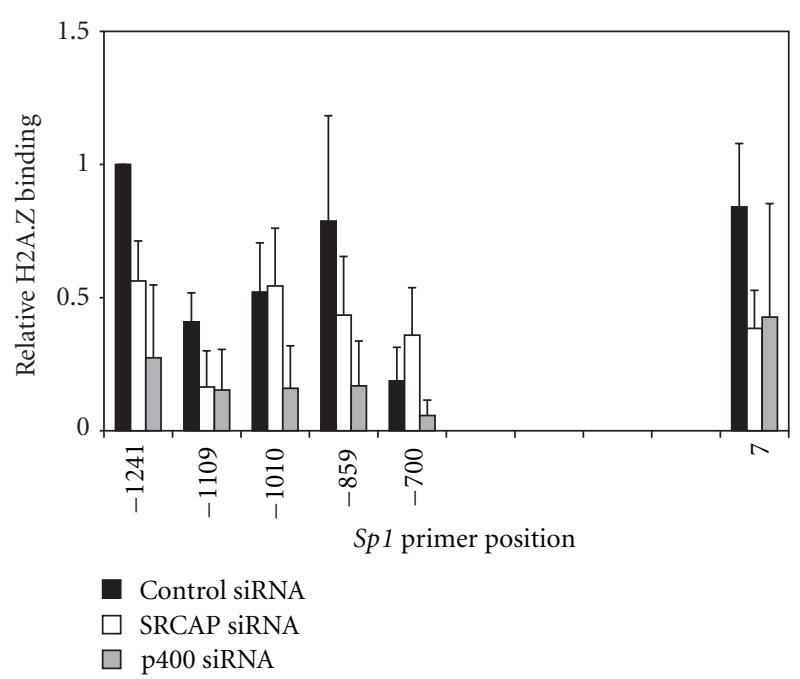

FIGURE 5: SRCAP and $\mathrm{p} 400$ regulate H2A.Z deposition equivalently at Sp1 promoter nucleosomes. 549 cells were transfected with control, SRCAP, or p400 siRNA and harvested 72 hours later. Mononucleosome ChIP assays were performed using anti-H2A.Z antibody and immunoprecipitated DNA was amplified by qPCR using the indicated primer sets (see Table S2). The amount of DNA amplified at each position is presented relative to the amount of DNA amplified at position -1241. The graph represents the mean result and standard error of three or more independent ChIP experiments.

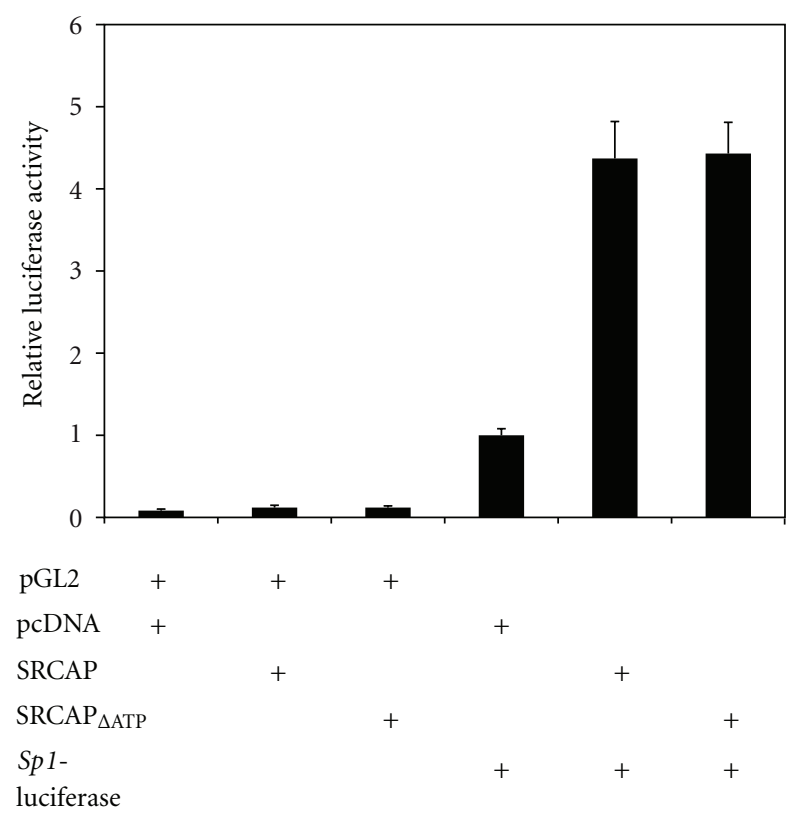

FIGURE 6: SRCAP mediates transcriptional activity independent of H2A.Z deposition. HeLa cells were transiently transfected with $300 \mu \mathrm{g}$ of $S p$-1-luciferase reporter gene plasmid or the control pGL2 luciferase reporter gene plasmid and, where indicated, with 1000 $\eta \mathrm{g}$ of plasmid expressing wild-type SRCAP or the $\mathrm{SRCAP}_{\triangle \mathrm{ATP}}$ mutant or the control vector pcDNA 3.1. The relative luciferase activity is reported compared to the luciferase activity observed in cells transfected with pGL2- luciferase and pcDNA 3.1. The graph represents the mean result and standard error of three or more independent transfection experiments. of SRCAP or p400 expression decreases overall H2A.Z deposition at promoters. Because of these collective observations, the role of the p400 and SRCAP complexes in regulating transcription has largely been attributed to the ability of each complex to deposit H2A.Z at promoters. In the case of the $p 21$ promoter, loss of H2A.Z deposition or knockdown of p400 (and subsequent loss of H2A.Z binding) results in activation of transcription. However, knockdown of SRCAP had equivalent effects on H2A.Z deposition as that seen with p400 knockdown, but did not increase transcription of the $p 21$ promoter. An interesting hypothesis raised by this finding is that deposition of H2A.Z at the $p 21$ promoter is not equivalent but is targeted by $\mathrm{p} 400$ or SRCAP to a distinct subset of nucleosomes.

In this report the loss of $\mathrm{p} 400$ or SRCAP resulted in $~ 50 \%$ decrease in deposition of H2A.Z into the same nucleosomes at $p 21$ promoter. This redundancy may result from an overlap in the nucleosomes targeted for H2A.Z deposition or may result from redistribution of H2A.Z-containing nucleosomes from unique sites where p400 or SRCAP mediates H2A.Z deposition to new positions across the promoter. In support of the latter, incorporation of H2A.Z has been reported to increase the mobility of nucleosomes [24]. Interestingly, knockdown of p400 or SRCAP did not effect the deposition of H2A.Z at several positions within the $p 21$ promoter, for example, positions -2557 and -2178 . This suggests that a third novel mechanism may exist for deposition of H2A.Z into some nucleosomes. Alternatively, the level of H2A.Z at any position is likely to result from equilibrium between two processes, H2A.Z incorporation and nucleosome turnover. It is therefore possible that despite the lack of $\mathrm{p} 400$ or SRCAP, the low rate of turnover of nucleosomes at these positions prevents a loss of $\mathrm{H} 2 \mathrm{~A}$. Z.

At the $S p 1$ promoter while loss of $\mathrm{p} 400$ also decreased H2A.Z deposition into all nucleosomes, the loss of SRCAP decreased H2A.Z deposition into all nucleosomes except those at positions -1010 and -700 . This observation suggests that in at least some promoters the method of H2A.Z deposition is a critical feature in maintaining H2A.Z deposition into some nucleosomes.

The presence of a large NFR was also noted in the Sp1 promoter region spanning positions -788 to -60 (Figure 4 ). Previous ChIP studies indicate these same regions are largely devoid of trimeH3K4, H2A.Z, and RNAPII and define the binding site for SRCAP [3]. Why SRCAP binds to this nucleosome free site is unclear but open chromatin may be required to allow binding of transcription factors that interact with CBP, which subsequently recruits SRCAP [19]. At other promoters, however, for example, FAD synthetase promoter, the binding sites for SRCAP and sites of H2A.Z directly coincide [3] suggesting other mechanisms might target the SRCAP to specific promoter sites.

Studies in S. cerevisiae have indicated that H2A.Z plays a role in nucleosome positioning and occupancy spanning the initiator region of the GAL1 gene [25]. This suggests that promoters enriched in H2A.Z have defined nucleosome locations compared with promoters that are not significantly enriched in H2A.Z. In addition, several studies indicate that H2A.Z containing chromatin is enriched in remodeling 
complexes (Swi/SNF, ISWI, and CHD) that facilitate movement of nucleosomes [26]. In support of this notion, recent studies also indicate that the presence of H2A.Z in nucleosomes facilitates remodeling activity by the ISWI family members. In contrast to the expectation raised by these observations, we found that despite the $\sim 50 \%$ decrease in deposition of H2A.Z following loss of p400 or SRCAP, the density of nucleosomes at the $p 21$ or $S p 1$ promoters is not altered. This suggests that at least at these promoters, the presence of H2A.Z does not facilitate chromatin-remodeling activity. One possible explanation for this observation is that $\mathrm{H} 2 \mathrm{~A} . \mathrm{Z}$ is a minor component of nucleosomes at these promoters and hence, cannot play a major role in determining remodeling activity or chromatin structure. A second possibility is a large percentage of the nucleosomes contain H2A.Z but the replacement with $\mathrm{H} 2 \mathrm{~A}$ does not have a significant role in determining nucleosome stability, remodeling, or overall chromatin structure. Either of these scenarios is more consistent with the role of $\mathrm{H} 2 \mathrm{~A} . \mathrm{Z}$ as a signaling molecule in which recruitment of general transcription factors is enhanced. Studies in human breast cancer cells suggest that p400-mediated deposition of H2A.Z at the estrogen receptor alpha-regulated gene, TFF, recruits FoxA1 to facilitate gene expression upon estrogen signaling [27]. This is further supported by studies in $S$. cerevisiae which demonstrate that H2A.Z-containing nucleosomes recruit Pol II and TBP to gene promoters $[28,29]$. The C-terminal region of $\mathrm{H} 2 \mathrm{~A} . \mathrm{Z}$ is critical for this activity and has been shown to function as an activating domain [30]. Substitution of this region by the equivalent $\mathrm{H} 2 \mathrm{~A}$ region does not rescue a loss of function mutation [28] nor does it rescue lethality in Drosophila melanogaster [31]. An acidic patch present in the C-terminal region of $\mathrm{H} 2 \mathrm{~A} . \mathrm{Z}$ compared to the $\mathrm{H} 2 \mathrm{~A}$ region likely facilitates these functions [32].

Although H2A.Z is important for transcription, a clear conclusion from these studies is that deposition of $\mathrm{H} 2 \mathrm{~A} . \mathrm{Z}$ at the $p 21$ and $S p 1$ promoters is not sufficient to explain the transcriptional activities of the p400 and SRCAP complexes. Recent evidence indicates the role each complex plays in regulating transcription is likely due to the structural differences between SRCAP and p400. The most notable difference is the presence of a SANT domain in p400, which is absent in SRCAP. The SANT domain of p400 has recently been shown to bind directly to the histone acetyltransferase domain of TIP60. This blocks enzymatic activity and the coactivator function of TIP60 in regulating basal $p 21$ gene expression via acetylated p53 [19]. Thus, loss of p400 activity mediates increased $p 21$ gene expression by two mechanisms: loss of TIP60 inhibition and loss of H2A.Z deposition. In the case of the Sp1 promoter, despite loss of H2A.Z deposition, loss of p400 expression did not alter transcription, suggesting that TIP60 does not play a critical role at this promoter. SRCAP also appears to use several mechanisms to regulate transcription. It also binds to the histone acetyltransferase CREB-binding protein (CBP) through a large spacer domain located between ATPase motifs IV and V that is not present in p400. In addition, CBP and SRCAP function synergistically to activate transcription [19]. The ability of SRCAP to activate transcription of the $S p 1$ promoter but not the $p 21$ promoter may be dependent on its ability to serve as a platform for recruitment of CBP. Consistent with the role of SRCAP to function as a recruitment platform, we found that the $\mathrm{SRCAP}_{\triangle \mathrm{ATP}}$ mutant retained the ability to activate transcription of the Sp1 promoter despite its inability to deposit H2A.Z. This mutant is not able to function in a transgenic fly model, implying that both the H2A.Z deposition and scaffolding activities of SRCAP are critical for its normal function in vivo. These results suggest that the deposition of H2A.Z does contribute to, but is not sufficient to describe, the transcriptional activity of SRCAP or p400.

In summary, our studies demonstrate that the p400 and SRCAP remodeling complexes have overlapping redundancy in targeting and deposition of H2A.Z into promoter nucleosomes. In addition, we demonstrate that while H2A.Z deposition may be a critical activity of these complexes, it is not sufficient to explain the effect the p400 and SRCAP complexes have on transcription of the $S p 1$ and p21 promoters.

\section{Materials and Methods}

4.1. Antibodies. The anti-SRCAP affinity-purified rabbit polyclonal antibody was generated against SRCAP as described in [15] and the anti-p400 antibody was raised in rabbits against the p400 C-terminal peptide (SSDSPSQQPKLQMRVPAVRLKTPTKPP). Other commercial antibodies were histone H2A.Z polyclonal (Abcam, ab4174), histone $\mathrm{H} 3$ polyclonal (Abcam, ab18262), and mouse monoclonal anti- $\beta$ actin antibody (Sigma, A5441).

4.2. Cell Culture. The human lung adenocarcinoma cell line, A549, and human cervical carcinoma, HeLa, (ATCC) were cultured in Dulbecco's modified Eagle's medium (Life Technologies) supplemented with $10 \%$ fetal bovine serum (Sigma) and 1\% penicillin/streptomycin (Life Technologies).

4.3. Reverse Transcription Quantitative Real-Time PCR (RT$q P C R)$. Total cellular RNAs were extracted with TRIzol reagent (Life Technologies) according to the manufacturer's protocol. Reverse transcription reactions were done as described in lab protocols [3] on $4 \mu \mathrm{g}$ of total RNAs, oligodT (Promega), and SuperScript II Reverse Transcriptase (Life Technologies) according to the manufacturer's protocol. cDNA levels were measured by RT-qPCR as in qPCR protocol with the following exceptions: the $\beta$-actin annealing temperature was $52^{\circ} \mathrm{C}$ for $30 \mathrm{~s}$ and quantification for 40 cycles, the $p 21$ annealing temperature was $60^{\circ} \mathrm{C}$ for $30 \mathrm{~s}$ and quantification for 40 cycles. Primer sequences for $p 21$ are listed in the Table S1(b).

4.4. Mononucleosome Preparation. Chromatin cross-linking and nuclei preparation was performed as described in [3]. The nuclei were resuspended in $500 \mu \mathrm{L}$ of $0.32 \mathrm{M}$ sucrose, $10 \mathrm{mM}$ HEPES, pH 7.9, $60 \mathrm{mM} \mathrm{KCl,} 2 \mathrm{mM}$ EDTA, $10 \mathrm{mM}$ sodium butyrate, $0.5 \mathrm{mM}$ DTT, $1 \mathrm{mM}$ PMSF, and supplemented with protease inhibitors (Roche Applied Science) and layered on top of $500 \mu \mathrm{L}$ of $30 \%$ sucrose $10 \mathrm{mM}$ HEPES, pH 7.9, $60 \mathrm{mM} \mathrm{KCl,} 2$ mM EDTA, $10 \mathrm{mM}$ sodium butyrate, 
$0.5 \mathrm{mM}$ DTT, $1 \mathrm{mM}$ PMSF supplemented with protease inhibitors (Roche Applied Science) and centrifuged at $500 \mathrm{xg}$ for 5 minutes at $4^{\circ} \mathrm{C}$. The pelleted nuclei were washed with micrococcal nuclease buffer (10 mM HEPES, pH 7.9, $60 \mathrm{mM} \mathrm{KCl}, 15 \mathrm{mM} \mathrm{NaCl}$, and $0.34 \mathrm{mM}$ sucrose) and resuspended in micrococcal nuclease buffer plus $10 \mathrm{mM}$ sodium butyrate, $3 \mathrm{mM} \mathrm{CaCl} 2, \mathrm{mM} 0.5 \mathrm{mM}$ DTT, and 0.6 Kunitz units of micrococcal nuclease (New England BioLabs) per microgram DNA at $37^{\circ} \mathrm{C}$ in a water bath for 11 minutes. The digestion was stopped on ice with the addition of EGTA $(10 \mathrm{mM})$ and nondigested chromatin was removed by centrifugation at $11,000 \times \mathrm{g}$ for 10 minutes at $4^{\circ} \mathrm{C}$. A portion of the supernatant was analyzed by fractionation on a sucrose gradient to determine the extent of digestion. A second portion was processed by Western blot analysis to verify that histones were present in the same DNA fraction containing mononucleosomes. A third portion was used to determine nucleosome density.

4.5. Quantitative Real-Time PCR ( $q P C R$ ). The amount of DNA corresponding to specific regions of the $p 21$ and $S p 1$ promoters present in the mononucleosome DNA preparations or in the nucleosome ChIP eluates was measured by quantitative real-time PCR (DNA Engine Opticon 2 System, Bio-Rad) with 2x fastStart SYBR Green master Mix (Roche Applied Science) according to protocols developed in our lab [3]. The reaction mixture consisted of $500 \eta \mathrm{M}$ of forward and reverse primer (see Tables S1(c) and S2), mononucleosomal DNA and the SYBR Green Master Mix (Roche Applied Science). The qPCR protocol was: $95^{\circ} \mathrm{C}$ for 5 minutes followed by three-step amplification (denaturation $95^{\circ} \mathrm{C}, 30$ seconds; annealing $60^{\circ} \mathrm{C}, 30$ seconds; extension $72^{\circ} \mathrm{C}, 40$ seconds) and quantification of DNA for 35 cycles.

To accurately determine the amount of promoter DNA within the nucleosome DNA sample, the standard curve method is used as described [3]. In this method, for each primer set, a series of amplification curves are generated using known amounts of genomic DNA (not nucleosome DNA). The equation for PCR kinetics is $N_{\mathrm{Ct}}=N_{\mathrm{O}} \times(\mathrm{eff})^{\mathrm{Ct}}$, where $N_{\mathrm{O}}$ is the initial amount of DNA in the sample, $N_{\mathrm{Ct}}$ is the amount of DNA at the threshold cycle, and eff is the PCR efficiency. This equation can be converted into a linear form Ct $=[-1 / \log ($ eff $)] \times \log N_{O}+\log \left(N_{\mathrm{Ct}}\right) / \log ($ eff). Therefore, a linear standard curve with a slope $=[-1 / \mathrm{Log}$ (eff)] can be constructed by plotting Ct values against the Log $N_{O}$ of the standards. As standards we use 10, 1, and $0.1 \mathrm{ng}$ of genomic DNA. DNA obtained from nucleosomes is not used as a standard, since it lacks promoter regions digested by micrococcal nuclease. For both the sample and standard curve reactions we typically obtain PCR efficiency of greater than $1.9(95 \%)$.

4.6. Knockdown of SRCAP or p400. Knockdown transfections were carried out according to lab protocols [3]. Cells were transfected using DharmaFECT1 (Dharmacon) siRNA transfection reagent according to the manufacturer's protocol to transfect A549 using 40nM of the Dicer substrate siRNA targeting: control, SRCAP, or p400 (see Supplemental Table S3, all siRNA came from IDT). Cells were harvested 72 hours after transfection for protein analysis, mononucleosomes preparation or RNA isolation.

4.7. Chromatin Immunoprecipitation. ChIP assays were performed according to the protocol developed in our lab [3].

4.8. Nucleosome Chromatin Immunoprecipitation. Mononucleosomes $(6 \mu \mathrm{g}$ DNA) were diluted to a final volume of $1 \mathrm{~mL}$ with Sonication buffer and precleared using $100 \mu \mathrm{L}$ of protein A/G-agarose beads ( $50 \%$ slurry) in PBS. The supernatant was cleared a second time using $200 \mu \mathrm{L}$ of protein $\mathrm{A} / \mathrm{G}$ beads (50\% slurry) that had been preblocked with nonfat milk (1\%), BSA (0.1\%) and normal rabbit IgG. The cleared ChIP lysate was then incubated for 18 hours with $12 \mu \mathrm{g}$ anti-H2A.Z antibody or $12 \mu \mathrm{g}$ normal rabbit IgG followed by additional 2-hour incubation with $50 \mu \mathrm{L}$ of protein $\mathrm{A} / \mathrm{G}$ beads $(50 \%$ slurry). The ChIP eluate was then obtained by washing the protein A/G beads and eluting the bound DNA as described above. Following reversal of cross-linking, protein digestion, and DNA purification, the DNA was resuspended in $100 \mu \mathrm{L}$ $\mathrm{dH}_{2} \mathrm{O}$.

4.9. Acid Extraction of Histones. A 459 nuclei were lysed in 5 volumes of $25 \mathrm{mM}$ HEPES (pH 7.8), $1.5 \mathrm{mM} \mathrm{MgCl}_{2}, 10 \mathrm{mM}$ $\mathrm{KCl}, 0.1 \%$ Nonidet P- $40,10 \mathrm{mM}$ sodium butyrate, $0.5 \mathrm{mM}$ DTT, $1.5 \mathrm{mM}$ PMSF, protease inhibitors (Roche Applied Science) and hydrochloric acid ( $0.2 \mathrm{M}$ final) for 30 minutes on ice. Sample was centrifuged $11,000 \mathrm{~g}$ for 10 minutes at $4^{\circ} \mathrm{C}$. The supernatant was dialyzed against $200 \mathrm{~mL}$ acetic acid

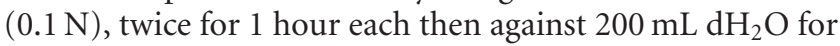
1 hour, 3 hours, and overnight. The sample was lyophilized to concentrate the proteins.

4.10. Transfection. HeLa cells were transfected with $300 \mu \mathrm{g}$ of the Sp1-luciferase or pGL2-luciferase expression plasmid and $1000 \eta \mathrm{g}$ of the plasmids expressing either SRCAP or $\mathrm{SRCAP}_{\triangle \mathrm{ATP}}$. The Sp1-luciferase plasmid was constructed by subcloning Sp1 promoter DNA $(-1241$ to +100$)$ into the KpnI and ZhoI sites of the pGL2 basic luciferase plasmid (Promega). The SRCAP (1-2971) or SRCAP SATP $_{\text {(K649R) }}$ expression plasmids were constructed as described $[18,24]$. Each transfection was adjusted to contain equal molar amounts of CMV promoter by use of the pcDNA3.1 Myc/His plasmid. Transfections were carried out using Lipofectamine (Life Technologies) according to the manufacturer's directions. Following an overnight incubation, cells were harvested and assayed for luciferase activity. The relative luciferase activity reported was performed in triplicate as described in [24].

\section{References}

[1] R. Faast, V. Thonglairoam, T. C. Schulz et al., "Histone variant H2A.Z is required for early mammalian development," Current Biology, vol. 11, no. 15, pp. 1183-1187, 2001.

[2] D. Rangasamy, I. Greaves, and D. J. Tremethick, "RNA interference demonstrates a novel role for H2A.Z in chromosome 
segregation," Nature Structural and Molecular Biology, vol. 11, no. 7, pp. 650-655, 2004.

[3] M. M. Wong, L. K. Cox, and J. C. Chrivia, "The chromatin remodeling protein, SRCAP, is critical for deposition of the histone variant H2A.Z at promoters," Journal of Biological Chemistry, vol. 282, no. 36, pp. 26132-26139, 2007.

[4] Z. Wang, C. Zang, J. A. Rosenfeld et al., "Combinatorial patterns of histone acetylations and methylations in the human genome," Nature Genetics, vol. 40, no. 7, pp. 897-903, 2008.

[5] N. Gévry, M. C. Ho, L. Laflamme, D. M. Livingston, and L. Gaudreau, "p21 transcription is regulated by differential localization of histone H2A.Z," Genes and Development, vol. 21, no. 15, pp. 1869-1881, 2007.

[6] I. Y. Chen, J. Lypowy, J. Pain et al., "Histone H2A.z is essential for cardiac myocyte hypertrophy but opposed by silent information regulator $2 \alpha$," Journal of Biological Chemistry, vol. 281, no. 28, pp. 19369-19377, 2006.

[7] A. Svotelis, N. Gévry, G. Grondin, and L. Gaudreau, "H2A.Z overexpression promotes cellular proliferation of breast cancer cells," Cell Cycle, vol. 9, no. 2, pp. 364-370, 2010.

[8] S. Hua, C. B. Kallen, R. Dhar et al., "Genomic analysis of estrogen cascade reveals histone variant H2A.Z associated with breast cancer progression," Molecular Systems Biology, vol. 4, article 188, 2008.

[9] A. Barski, S. Cuddapah, K. Cui et al., "High-resolution profiling of histone methylations in the human genome," Cell, vol. 129, no. 4, pp. 823-837, 2007.

[10] D. E. Schones, K. Cui, S. Cuddapah et al., "Dynamic regulation of nucleosome positioning in the human genome," Cell, vol. 132, no. 5, pp. 887-898, 2008.

[11] Y. J. Park, P. N. Dyer, D. J. Tremethick, and K. Luger, "A new fluorescence resonance energy transfer approach demonstrates that the histone variant $\mathrm{H} 2 \mathrm{AZ}$ stabilizes the histone octamer within the nucleosome," Journal of Biological Chemistry, vol. 279, no. 23, pp. 24274-24282, 2004.

[12] A. A. Thambirajah, D. Dryhurst, T. Ishibashi, A. Li, A. H. Maffey, and J. Ausió, "H2A.Z stabilizes chromatin in a way that is dependent on core histone acetylation," Journal of Biological Chemistry, vol. 281, no. 29, pp. 20036-20044, 2006.

[13] C. Jin and G. Felsenfeld, "Nucleosome stability mediated by histone variants H3.3 and H2A.Z," Genes and Development, vol. 21, no. 12, pp. 1519-1529, 2007.

[14] C. Jin, C. Zang, G. Wei et al., "H3.3/H2A.Z double variantcontaining nucleosomes mark 'nucleosome-free regions' of active promoters and other regulatory regions," Nature Genetics, vol. 41, no. 8, pp. 941-945, 2009.

[15] A. Thakar, P. Gupta, T. Ishibashi et al., "H2A.Z and H3.3 histone variants affect nucleosome structure: biochemical and biophysical studies," Biochemistry, vol. 48, no. 46, pp. 1085210857, 2009.

[16] D. D. Ruhl, J. Jin, Y. Cai et al., "Purification of a human SRCAP complex that remodels chromatin by incorporating the histone variant H2A.Z into nucleosomes," Biochemistry, vol. 45, no. 17 , pp. 5671-5677, 2006.

[17] Y. Cai, J. Jin, C. Tomomori-Sato et al., "Identification of new subunits of the multiprotein mammalian TRRAP/TIP60containing histone acetyltransferase complex," Journal of Biological Chemistry, vol. 278, no. 44, pp. 42733-42736, 2003.

[18] Y. Cai, J. Jin, L. Florens et al., "The mammalian YL1 protein is a shared subunit of the TRRAP/TIP60 histone acetyltransferase and SRCAP complexes," Journal of Biological Chemistry, vol. 280, no. 14, pp. 13665-13670, 2005.
[19] M. A. Monroy, D. D. Ruhl, X. Xu, D. K. Granner, P. Yaciuk, and J. C. Chrivia, "Regulation of cAMP-responsive elementbinding protein-mediated transcription by the SNF2/SWIrelated protein, SRCAP," Journal of Biological Chemistry, vol. 276, no. 44, pp. 40721-40726, 2001.

[20] J. H. Park, X. J. Sun, and R. G. Roeder, “The SANT domain of p400 ATPase represses acetyltransferase activity and coactivator function of TIP60 in basal p21 gene expression," Molecular and Cellular Biology, vol. 30, no. 11, pp. 2750-2761, 2010.

[21] P. D. Hartley and H. D. Madhani, "Mechanisms that specify promoter nucleosome location and identity," Cell, vol. 137, no. 3, pp. 445-458, 2009.

[22] R. M. Raisner, P. D. Hartley, M. D. Meneghini et al., "Histone variant H2A.Z Marks the 5' ends of both active and inactive genes in euchromatin," Cell, vol. 123, no. 2, pp. 233-248, 2005.

[23] J. C. Eissenberg, M. Wong, and J. C. Chrivia, "Human SRCAP and Drosophila melanogaster DOM are homologs that function in the Notch signaling pathway," Molecular and Cellular Biology, vol. 25, no. 15, pp. 6559-6569, 2005.

[24] A. Flaus, C. Rencurel, H. Ferreira, N. Wiechens, and T. OwenHughes, "Sin mutations alter inherent nucleosome mobility," EMBO Journal, vol. 23, no. 2, pp. 343-353, 2004.

[25] B. Guillemette, A. R. Bataille, N. Gévry et al., "Variant histone H2A.z is globally localized to the promoters of inactive yeast genes and regulates nucleosome positioning," PLoS Biology, vol. 3, no. 12, article 384, pp. 1-11, 2005.

[26] J. A. Goldman, J. D. Garlick, and R. E. Kingston, "Chromatin remodeling by imitation switch (ISWI) class ATP-dependent remodelers is stimulated by histone variant H2A.Z," Journal of Biological Chemistry, vol. 285, no. 7, pp. 4645-4651, 2010.

[27] N. Gévry, S. Hardy, P. É. Jacques et al., "Histone H2A.Z is essential for estrogen receptor signaling," Genes and Development, vol. 23, no. 13, pp. 1522-1533, 2009.

[28] M. Adam, F. Robert, M. Larochelle, and L. Gaudreau, "H2A.Z is required for global chromatin integrity and for recruitment of RNA polymerase II under specific conditions," Molecular and Cellular Biology, vol. 21, no. 18, pp. 6270-6279, 2001.

[29] Y. Wan, R. A. Saleem, A. V. Ratushny et al., "Role of the histone variant H2A.Z/Htz1p in TBP recruitment, chromatin dynamics, and regulated expression of oleate-responsive genes," Molecular and Cellular Biology, vol. 29, no. 9, pp. 2346-2358, 2009.

[30] M. Larochelle and L. Gaudreau, "H2A.Z has a function reminiscent of an activator required for preferential binding to intergenic DNA," EMBO Journal, vol. 22, no. 17, pp. 45124522, 2003.

[31] M. J. Clarkson, J. R. E. Wells, F. Gibson, R. Saint, and D. J. Tremethick, "Regions of variant histone His2AvD required for Drosophila development," Nature, vol. 399, no. 6737, pp. 694697, 1999.

[32] R. K. Suto, M. J. Clarkson, D. J. Tremethick, and K. Luger, "Crystal structure of a nucleosome core particle containing the variant histone H2A.Z," Nature Structural Biology, vol. 7, no. 12, pp. 1121-1124, 2000. 

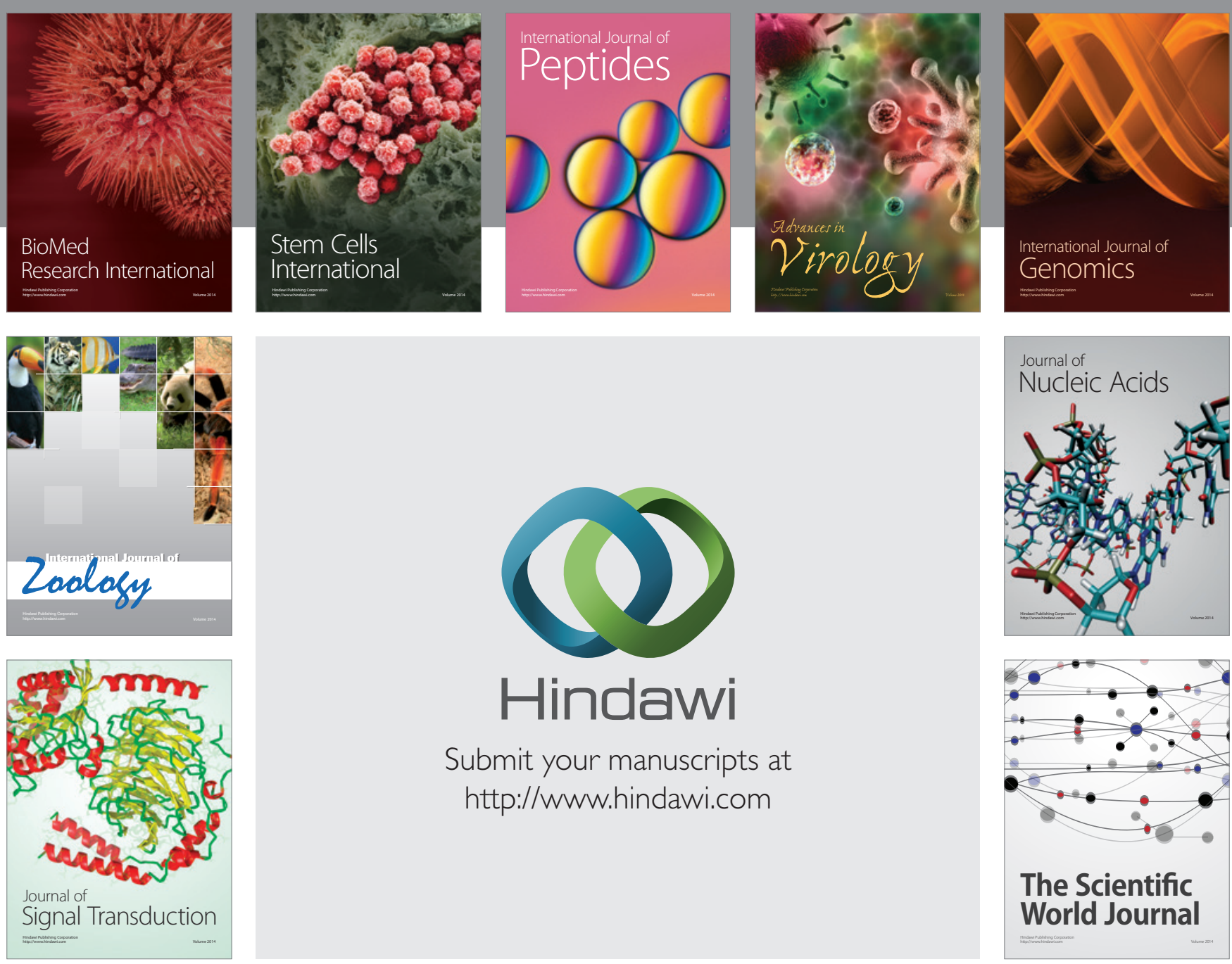

Submit your manuscripts at

http://www.hindawi.com
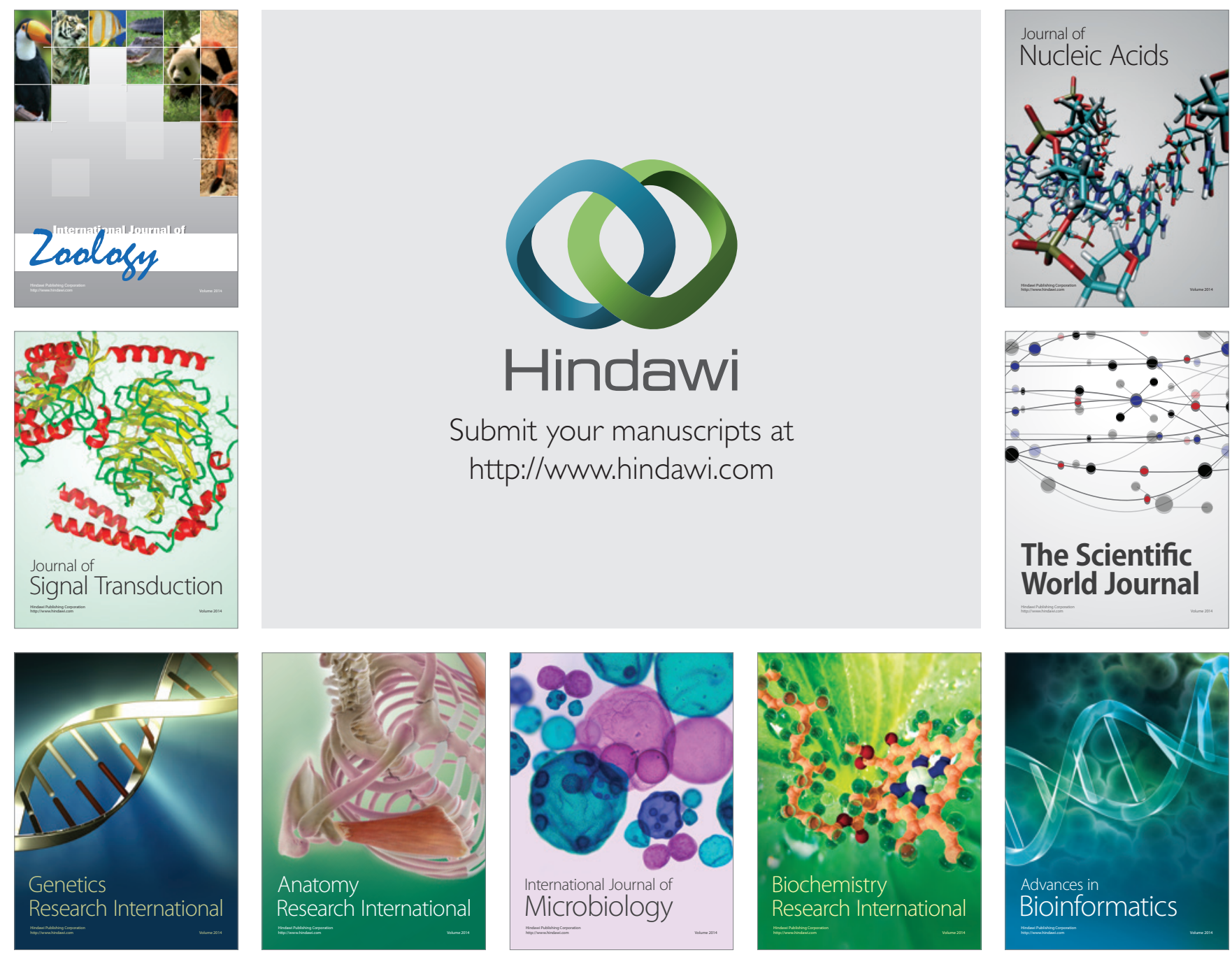

The Scientific World Journal
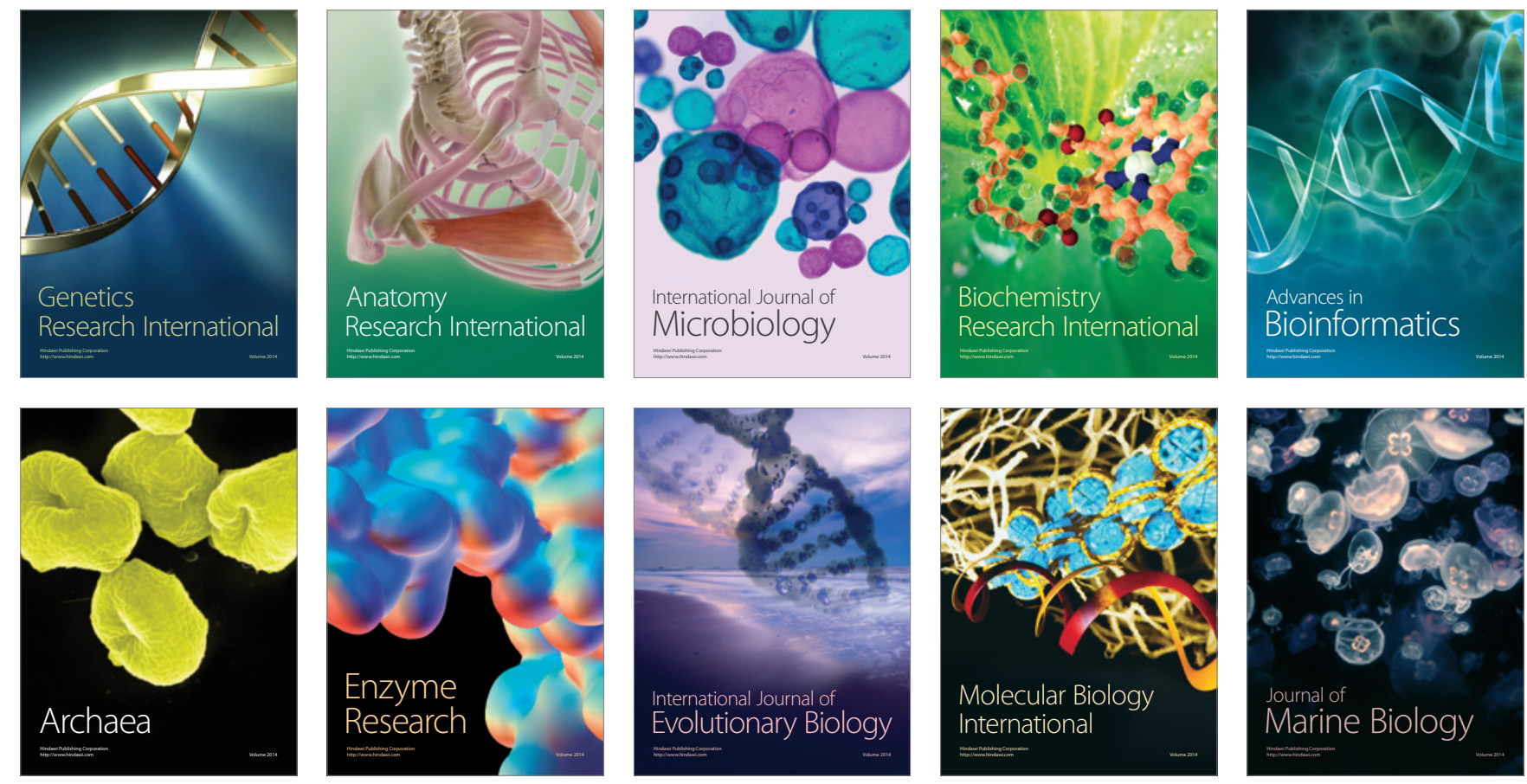